\title{
Use of Acellular Dermal Matrix for Urethroplasty Coverage in Proximal Hypospadias Repair: a Pilot Study
}

\author{
Defu Lin · Guannan Wang · Hongcheng Song · Yanchao Qu • \\ Pei Liu · Haiyan Liang · Shuai Xu · Shuofan Chen · Weiping Zhang · \\ Yannan Zhao $\cdot$ Bing Chen $\cdot$ Ning Sun (1)
}

Received: January 13, 2020 / Published online: February 15, 2020

(C) The Author(s) 2020

\section{ABSTRACT}

Introduction: The complication rates of proximal hypospadias, especially fistula, are much higher than those of distal hypospadias. Urethral coverage is an effective method for reducing fistulas. Acellular dermal matrix (ADM) has been shown to exhibit structural compatibility and biocompatibility, both of which promote tissue healing.

Methods: The present non-randomized study evaluated the efficiency, feasibility, and safety of using ADM for urethroplasty coverage in patients with proximal hypospadias. This prospective study enrolled 35 patients (age range

Enhanced Digital Features To view enhanced digital features for this article go to https://doi.org/10.6084/ m9.figshare.11798895.

Defu Lin and Guannan Wang contributed equally to this work.

D. Lin · G. Wang · H. Song · Y. Qu · P. Liu .

H. Liang · S. Xu · S. Chen · W. Zhang · N. Sun ( $\triangle)$ Department of Urology, Beijing Children's Hospital Affiliated to Capital Medical University, National Center for Children's Health, Beijing 100045, China e-mail: doctorsn@163.com

\section{Y. Zhao · B. Chen $(\bowtie)$}

State Key Laboratory of Molecular Developmental Biology, Institute of Genetics and Developmental Biology, Chinese Academy of Sciences, Beijing 100190, China

e-mail: bchen@genentica.ac.cn
15-60 months) with proximal hypospadias who underwent operation between September 2018 and March 2019 at Beijing Children's Hospital (Beijing, China). Urethroplasties were performed by the transverse preputial island flap (TPIF) technique. ADM was applied and sutured over the urethroplasty as an additional covering layer. Patient outcomes were compared with those of 80 non-matched control patients with proximal hypospadias who underwent the same procedure, with dartos as a covering layer.

Results: During a median follow-up of 11.56 months (range 9-15 months), urethral fistula occurred in six patients $(17.1 \%)$ in the ADM group and 28 patients (35\%) in the dartos group. Superficial wound infection was observed in six patients $(17.1 \%)$ in the ADM group and 10 patients (12.5\%) in the dartos group. One patient in the ADM group had diverticulum, compared with five patients $(6.25 \%)$ in the dartos group. Meatal stenosis and urethral stricture were observed in four patients $(11.4 \%)$ in the ADM group and six patients (7.5\%) in the dartos group; all of these complications were treated conservatively. No glans dehiscence was observed in either group.

Conclusion: Use of ADM may be a safe and efficient covering technique to provide an additional coverage layer for proximal hypospadias repair, thereby reducing the incidence of fistula formation, especially among patients who have poor-quality covering materials. 
Keywords: Acellular dermal matrix; Fistula; Proximal hypospadias; Urethral coverage; Urology

\section{Key Summary Points}

Why carry out this study?

The complication rates of proximal hypospadias, especially fistula, are much higher than those of distal hypospadias

Acellular dermal matrix (ADM), as a urethral covering layer, has been shown to exhibit structural compatibility and biocompatibility, both of which promote tissue healing

What was learned from the study?

Use of this biomaterial resulted in reduced incidence of urethrocutaneous fistula after urethroplasty in patients with severe hypospadias

This approach might constitute an effective strategy for hypospadias repair surgery

\section{INTRODUCTION}

Hypospadias is one of the most common congenital urinary malformations, with an incidence of 1 in 200-300 male births [1]. Proximal hypospadias is more difficult to treat than distal hypospadias. In particular, proximal hypospadias involves a longer urethral defect, with the meatus opening at the root of the penis or perineum. The incidence of complications following hypospadias surgery is related to the complexity of the repair: it is generally less than $2 \%$ with the meatal advancement and glanuloplasty incorporated technique, 5-10\% with meatal-based flaps [2]. Complications from proximal hypospadias repair range from $6 \%$ to $30 \%$ depending on the severity of the defect, the surgical technique utilized, and the experience of the surgeon [3].
A covering layer between the neo-urethra and the reconstructed skin considerably reduces fistula formation and the overall incidence of complications [4]. The use of neo-urethra-covering materials is regarded as an effective procedure to avoid urethrocutaneous fistula, which could involve the use of preputial skin grafts, bladder epithelium, buccal mucosa, tunica vaginalis, small intestinal submucosa, and tissue engineered materials [5]. Of these covering materials, the dartos is widely regarded as the gold standard for neo-urethral covering in proximal hypospadias repair due to its durability and excellent engraftment properties [6]. However, some patients with hypospadias have lost effective autogenic covering tissues as a result of circumcision procedures or multiple surgeries. Therefore, a readily available material that can promote cellular in-growth and neovascularity would be an ideal substance for use in proximal hypospadias repair.

Recently, acellular dermal matrix (ADM) was used successfully for treatment of patients with urethral stricture disease and bladder regeneration. The material originates from bovine fullthickness skin and is prepared through a multistep process that leads to the removal of cellular components; the resulting tissue matrix consists of collagen type I [7]. Collagen is a primary protein in connective tissue and the most abundant protein in the human body, comprising $30 \%$ of the overall protein content [8]. ADM is reportedly biocompatible, with weak antigenicity and sufficient biodegradability; it has been used to guide tissue growth in several experimental animal and clinical studies $[9,10]$. ADM provides an anchorage scaffold for cell adhesion, migration, and differentiation [11]. To the best of our knowledge, ADM has not yet been used as a covering layer for hypospadias repair.

Here, we hypothesized that ADM could be used to provide an interposition layer that enhances the structure surrounding the neourethral, thereby reducing the incidence of fistula formation.

This preliminary study compared outcomes between ADM and dartos used as covering materials in patients undergoing proximal hypospadias repair. 


\section{METHODS}

\section{Patient Selection}

This non-randomized study enrolled 35 patients (age range 15-60 months) with proximal hypospadias who underwent ADM operations between September 2018 and March 2019 at Beijing Children's Hospitals (Beijing, China). Patient outcomes were compared with those of 80 non-matched control patients with proximal hypospadias who underwent the same procedure with dartos as a covering layer. The main inclusion criteria of the ADM group and dartos group were as follows: (i) primary male patients with proximal hypospadias with severe chordee $\left(>30^{\circ}\right)$; (ii) hypospadias repair by transverse preputial island flap (TPIF) technique; (iii) written informed consent from patients' parents following explanation of the nature of hypospadias and various methods of treatment; (iv) ability and willingness to regularly visit the hospital for follow-up during the study period. The main exclusion criteria of the ADM group and dartos group were as follows: (i) presence of distal hypospadias, recurrent hypospadias, and/ or disorder of sex development, or receipt of preoperative topical androgen therapy; (ii) history of life-threatening allergic or immune-mediated reaction; (iii) clinically significant abnormalities in routine laboratory examinations; (iv) no use of the TPIF technique in hypospadias repair; (v) participation in any other clinical trials within 3 months before enrollment in this study; (vi) poor compliance with study requirements. The clinical study was approved by the ethics committee of Beijing Children's Hospital (IEC-C-006-A03-V.05). All procedures performed in studies involving human participants were performed in accordance with the ethical standards of Beijing Children's Hospital and with the 1964 Helsinki declaration and its later amendments or comparable ethical standards. Informed consent was obtained from parents to participate in the study and publish the results.

\section{Preparation and Biological Safety Evaluation of ADM}

ADM (collagen type I) was derived from bovine skin and prepared by Zhenghai Biotechnology (Shandong, China); it exhibited a porous stereoscopic structure, as previously reported [12]. The ADM was sterilized by $12 \mathrm{kGy} \mathrm{Co}^{60}$ irradiation. The surface morphology of collagen membranes was analyzed using a scanning electron microscope (Model S-2500; Hitachi), as well as by hematoxylin and eosin staining [13].

Acute toxicity, chronic toxicity, pyrogen reaction, allergen detection, genetic toxicity, intradermal irritation, cytotoxicity, hemolytic toxicity, and degradability characteristics of the ADM were evaluated by the National Institute of Food and Drug Control, in accordance with the Chinese Criterion of Medical Device GB16886.

\section{Surgical and ADM Transplantation Procedures}

All patients underwent hypospadias repair using the TPIF technique. Prophylactic preoperative intravenous antibiotic cefamandole $(50 \mathrm{mg} / \mathrm{kg}$ ) was routinely administered to all patients, 30 min prior to surgery. General anesthesia was induced in all patients; they were then placed in the supine position. After sterilization and draping had been performed, a glans stay suture was placed in the midline along the long axis of the penis. After complete degloving and excision of fibrotic tissue in the ventral aspect had been performed, the urethral plate was sectioned transversely at the position with severe chordee. Dorsal midline plication was performed to completely correct the chordee and rotational deformity. The dorsal preputial island flap was harvested and tubularized with an 8F silicon catheter (Fig. 1a, b). The length of the reconstructed tube was meticulously measured with a vernier caliper. The new tubularized urethral tube was sutured to the proximal urethral meatus and the tip of the glans (Fig. 1c). The single-layer pedicle dartos flap was used to cover the neo-urethra (Fig. 1d). An ADM sheet was trimmed to fit the length and width 

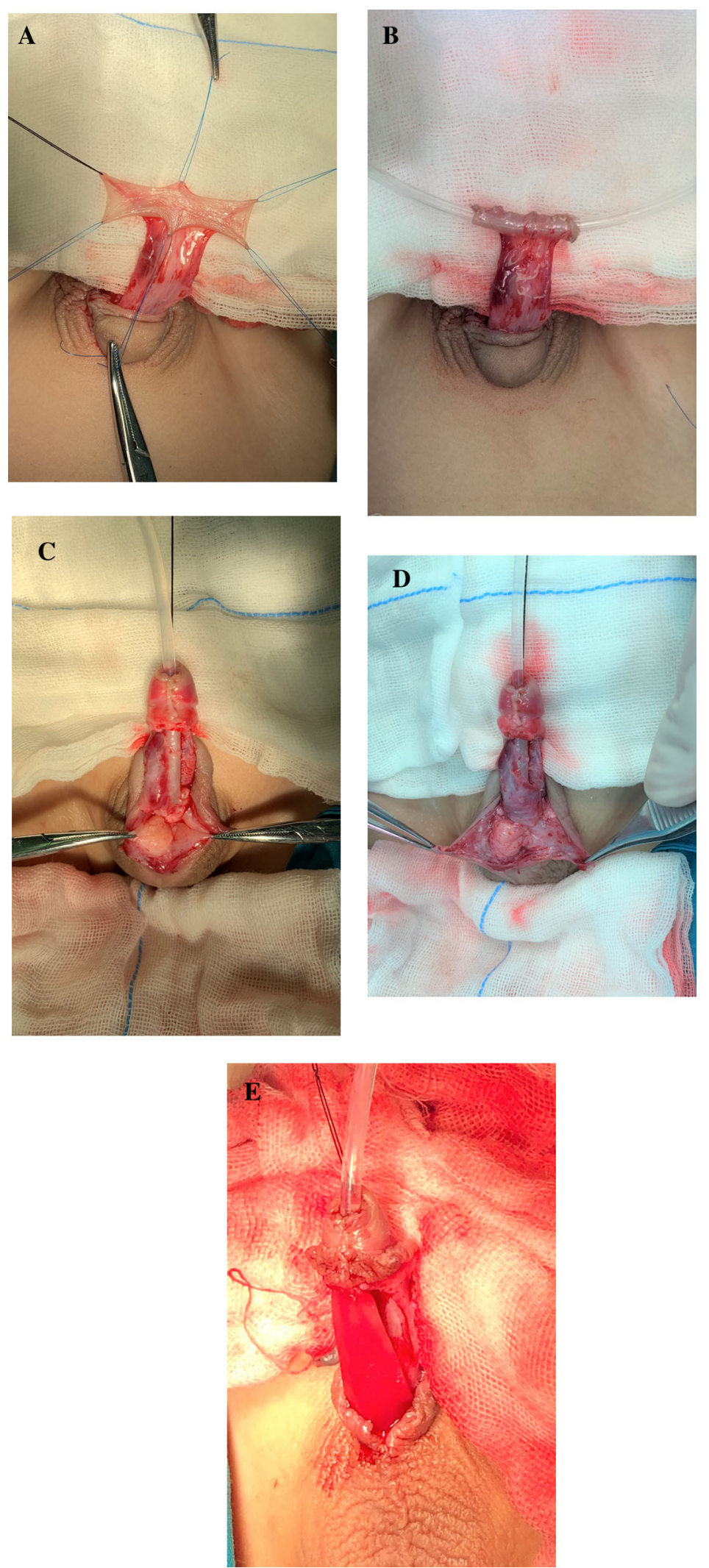
4 Fig. 1 Surgical procedure of transverse preputial island flap technique with pedicle dartos. a The dorsal preputial island flap was harvested. b The neo-urethra was tubularized with an $8 \mathrm{~F}$ silicon catheter. $\mathbf{c}$ The new tubularized urethral tube was sutured with the proximal urethral meatus and the tip of the glans. $\mathbf{d}$ The pedicle dartos flap was used to cover the neo-urethra. e The ADM coverage was applied over urethroplasty and secured by Mono-vicryl $6 / 0$

of the neo-urethral. The average width and length of the $\mathrm{ADM}$ were $1.5 \mathrm{~cm}$ and $3 \mathrm{~cm}$, respectively. ADM was applied after completing single-layer dartos covering and secured by Mono-vicryl 6/0 (Ethicon, USA) (Fig. 1e); the skin was then sutured directly without any other covering layers.

The control group included 80 patients with proximal hypospadias of similar severity; for all control patients, surgery was performed by the same surgeon and postoperative care was identical to that provided for patients in the ADM group. For control patients, a single-layer pedicle dartos flap was used to cover the urethroplasty.

For all patients in this study, the wound was covered with soft permeable dressing that allowed absorption of any minor bleeding from the wound. The compressive dressing was removed for all patients on the fifth postoperative day. An indwelling transurethral catheter was kept for 21 days to reduce the incidence of urethral stricture. The urinary stream was evaluated in all patients after catheter removal.

\section{Follow-Up}

All patients underwent follow-up for a maximum of 15 months; the mean follow-up duration was 11.56 months (range 9-15 months). Outpatient clinical assessment was performed at the time of catheter removal, as well as at 6 and 9 months after surgery. The outcomes of the patients were assessed by two of the authors with respect to operative time, postoperative complications, urinary stream, and cosmetic appearance. Complications were defined as fistula, urinary stricture, glandular dehiscence, and tissue infection. Cosmetic outcomes were evaluated by parents and based on the Pediatric Penile Perception Score (PPPS); the cosmetic parameters evaluated were normal meatus appearance and parental satisfaction. Success was defined as a lack of complications requiring re-intervention, with good cosmetic results. A functional outcome was defined as the presence of a normal voiding stream [14].

\section{Statistical Analysis}

All statistical analyses were performed using SPSS Statistics software version 24.0 (IBM, USA). Categorical data between the two groups were compared using the chi-square of Fisher's exact test. A $P$ value of less than 0.05 was considered statistically significant.

\section{RESULTS}

\section{Structure of Acellular Dermal Matrix}

We examined the histological structure of ADM by hematoxylin and eosin staining (Fig. 2a). The degree of cell removal and state of the dermal scaffold could be clearly determined. No epidermis, cells, or appendage organs were present in the ADM. ADM consisted of a dermal scaffold comprising porous collagen fibers. Scanning electron microscopy results are shown in Fig. 2b-d. Fiber bundles and porous fiber structure were detected. The biological safety of the ADM (Fig. 2e) was approved by the National Institute of Food and Drug Control, indicating that it met the Chinese Criterion of Medical Device GB16886.

\section{ADM Reduced the Incidence of Fistula}

In total, 115 patients with proximal hypospadias met the inclusion criteria (35 in the ADM group and 80 in the dartos group) from September 2018 to March 2019 (Table 1). The mean patient age at the time of surgery was 19 months (range 15-60 months). No patients had a history of hypospadias repair. The two groups were similar in terms of demographic 

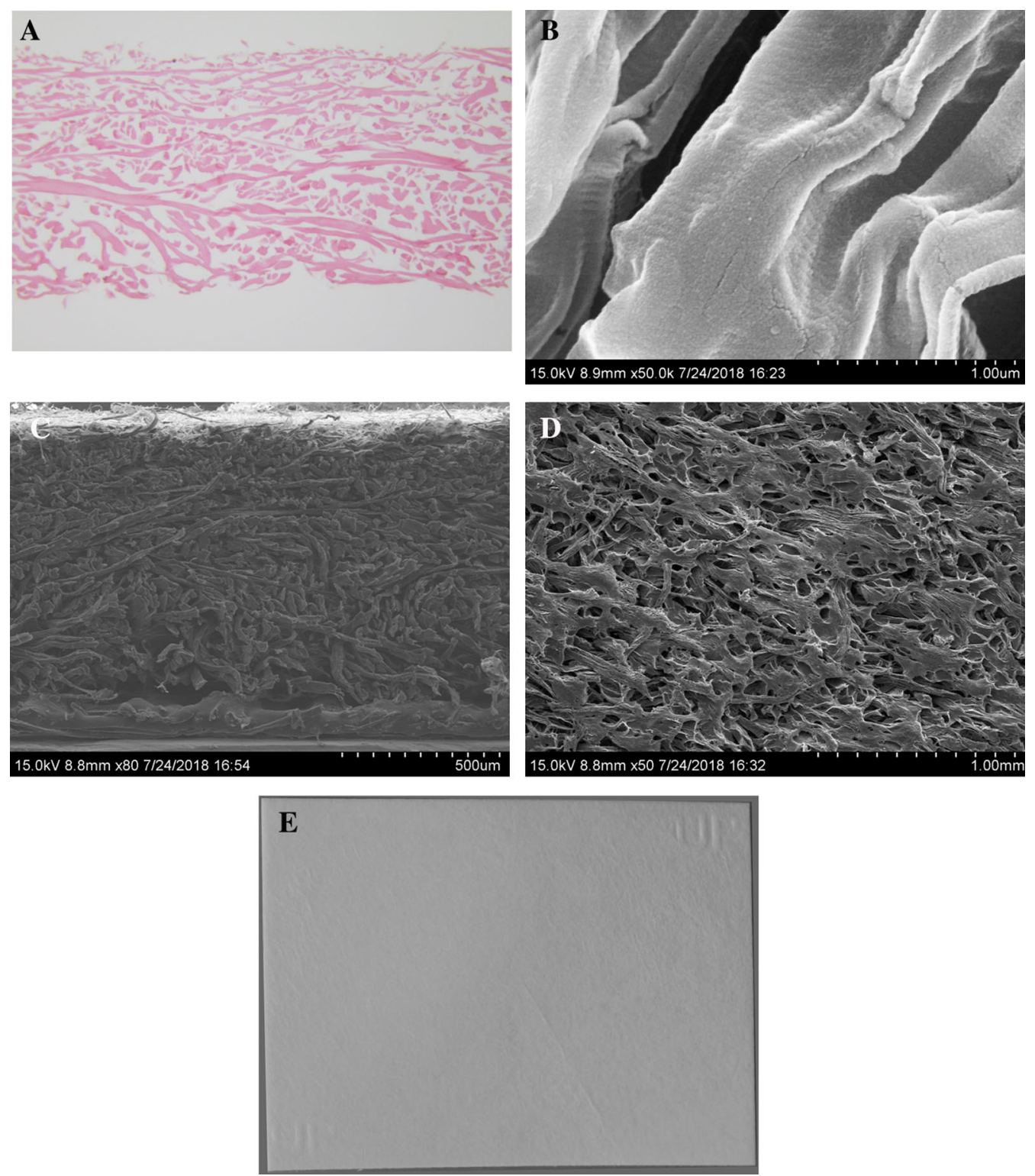

Fig. 2 Appearance and structure of acellular dermal matric for urethral coverage. a H\&E staining showed no epidermis, cells, or appendage organs present in ADM.

and clinical characteristics. Urethrocutaneous fistula was the most common complication within 6 months after surgery, occurring in six patients $(17.1 \%)$ in the ADM group and 28 patients $(35.0 \%)$ in the dartos group; the difference between groups was statistically significant $(P=0.04)$. No further fistulas were diagnosed at 6-month follow-up visit. b-d Scanning electron microscopy showed ADM with 2-200 $\mu \mathrm{m}$ pore size. e ADM was $3.0 \mathrm{~cm}$ long, $2.0 \mathrm{~cm}$ wide, and $0.30-0.69 \mathrm{~mm}$ thick

\section{ADM Urethral Coverage Does Not Increase Operative Time}

The mean operative time in the ADM group was $92 \mathrm{~min}$, which was slightly greater than that in the dartos group ( $88 \mathrm{~min})$. Trimming of the ADM was simple and coverage of the neo- 
Table 1 Characteristic of the ADM group and the dartos group

\begin{tabular}{|c|c|c|c|}
\hline Characteristic & $\begin{array}{l}\text { ADM } \\
\text { group } \\
n=35\end{array}$ & $\begin{array}{l}\text { Dartos } \\
\text { group } \\
n=\mathbf{8 0}\end{array}$ & $P$ value \\
\hline Age (months) & 16.25 & 20.2 & 0.465 \\
\hline $\begin{array}{l}\text { Mean operative duration } \\
\quad(\min )\end{array}$ & 92 & 88 & 0.372 \\
\hline $\begin{array}{l}\text { Amount of blood loss } \\
(\mathrm{ml})\end{array}$ & 4.9 & 4.6 & 0.518 \\
\hline Operative technique & TPIF & TPIF & \\
\hline Foley catheter & $8 \mathrm{~F}$ & $8 \mathrm{~F}$ & \\
\hline $\begin{array}{l}\text { Indwelling time of } \\
\text { catheter (days) }\end{array}$ & 21 & 21 & 0.316 \\
\hline $\begin{array}{l}\text { Mean length of urethral } \\
\text { defect }(\mathrm{cm})\end{array}$ & 29.5 & 30.2 & 0.565 \\
\hline
\end{tabular}

TPIF transverse preputial island flap technique

urethra could be performed without an increase in surgical time.

\section{ADM Was Safer for Hypospadias Repair as Urethral Coverage}

Six patients (17.1\%) exhibited wound infection in the ADM group, while 10 patients (12.5\%) exhibited wound infection in the dartos group $(P=0.34)$; the difference was likely related to improvement in debridement and dressing change. No surgery-related complications were identified intraoperatively; moreover, no fever, allergic reaction, or foreign body reaction occurred. No glans dehiscence was observed in any of the patients in either group.

\section{Complications}

A total of 17 complications (47.2\%) were reported in the ADM group, while 49 complications $(61.2 \%)$ were reported in the dartos group; the difference was statistically significant $(P=0.04)$ (Table 2). Out of 35 operations in the ADM group, $17(47.2 \%)$ had a postoperative complication of any kind. The majority (10 patients, $58.8 \%$ of all complications) had minor complication (Clavien-Dindo grade I or II). Seven patients (41.2\%) had grade IIIb, requiring a second-phase hypospadias repair after 6-12 months of the first operation. Out of 80 operations in the dartos group, 49 (61.2\%) had a postoperative complication. The majority (33 patients, $67.3 \%$ of all complications) had grade IIIb. Sixteen patients (32.7\%) had grade I or II. No Clavien-Dindo higher than grade IV was found in both groups. Four urethral strictures were detected in the ADM group, while six were detected in the dartos group $(P=0.36)$. Urethral strictures were improved by transurethral dilation and placement of an indwelling catheter for 3 weeks. A slit-like meatus on the tip of the glans was achieved in most patients. Patients' parents were asked to score the

Table 2 Complications of the ADM group and the dartos group

\begin{tabular}{lcccc}
\hline Complications & $\begin{array}{l}\text { ADM group } \\
\boldsymbol{n}=\mathbf{3 5}\end{array}$ & $\begin{array}{l}\text { Dartos group } \\
\boldsymbol{n}=\mathbf{8 0}\end{array}$ & $\begin{array}{l}\text { Total } \\
\boldsymbol{n}=\mathbf{1 2 5}\end{array}$ & $\boldsymbol{P}$ value \\
\hline Fistula & $6(17.1 \%)$ & $28(35.0 \%)$ & $34(29.6 \%)$ & 0.04 \\
Wound infection & $6(17.1 \%)$ & $10(12.5 \%)$ & $16(12.8 \%)$ & 0.34 \\
Glans dehiscence & $0(0 \%)$ & $0(0 \%)$ & $0(0 \%)$ & \\
Urethral stricture & $4(11.4 \%)$ & $6(7.5 \%)$ & $10(8.0 \%)$ & 0.36 \\
Diverticulum & $1(2.9 \%)$ & $5(6.25 \%)$ & $6(4.8 \%)$ & 0.41 \\
Total & $17(47.2 \%)$ & $49(61.2 \%)$ & $66(52.8 \%)$ & 0.04 \\
\hline
\end{tabular}


appearance as good, acceptable, or bad. The cosmetic result was reported as good in $76.7 \%$ of patients in the ADM group and $78.5 \%$ of patients in the dartos group.

\section{DISCUSSION}

Because $\mathrm{ADM}$ is a natural tissue matrix, it has many potential advantages compared with synthetic scaffold materials; upon implantation, ADM should be infiltrated and remodeled by host cells into long-lived functional tissue [15]. The structure of the extracellular matrix is highly conserved across different species; usable collagenous matrices can be derived from xenogenic sources [16]. ADM is reportedly biodegradable and does not cause significant inflammation, tissue fibrosis, or foreign-body reaction [17]. Current Food and Drug Administration-approved uses include dentistry, orthopedics, plastic surgery, and oral surgery. Uses for ADM have substantially increased in urological surgery in recent years, including bladder regeneration, cloacal malformation, and urethral surgery. The principles involved in the ingrowth of host tissue include contributions from host or matrix growth factors, re-epithelialization, vascularization, and tissue de-differentiation [18]. ADM is non-toxic, biocompatible, and self-degrading; moreover, it has provided an optimal environment for host cell repopulation.

The most common complication of hypospadias surgery is fistula formation. In the present study, we used ADM for coverage of the neo-urethral to reduce fistula formation on the basis of the results of prior clinical and experiment studies showing that ADM could improve wound healing. There is increasing evidence that collagen scaffolds, which support reconstruction of the microenvironment, can guide cell regeneration. Our previous study showed that collagen scaffolds can promote tissue regeneration and improve neo-urethra function in a canine model of extensive urethral defects [19]. Moreover, el-Kassaby et al. [6] reported that acellular matrix would be suitable for use in surgical treatment of early strictures with an apparently healthy urethral bed and minimal spongiofibrosis; this suitability is largely because of the "off-the-shelf nature" of the material, which eliminates the need for a tissue harvesting procedure that is commonly used in buccal mucosa-derived repair. ADM can be prepared during the urethroplasty procedure and used immediately. Chen et al. [13] used collagen membranes and collagen-binding basic fibroblast growth factor (CBD-bFGF) for bladder regeneration; they showed that this system could improve regeneration. Shi et al. [20] demonstrated that a bladder acellular matrix loaded with a CBD-bFGF could promote regeneration of bladder smooth muscle, vasculature, and nerves; in addition, it could improve the neobladder function.

In the present study, the incidence of fistula formation was significantly lower in the ADM group than in the dartos group. This result is similar to the findings reported for fibrin glues. As a covering material, fibrin glue is one of the main biomaterials promoting urethral healing in hypospadias repair surgery. Gopal et al. [21] observed that fistula formation was significantly lower in their series: rates of fistula formation were $15 \%$ and $31 \%$, respectively, for the fibrin glue and non-fibrin glue groups following treatment of proximal penile hypospadias. Improving wound healing and applying urethral covering are two main common characteristics of ADM and fibrin glue [22]. We hypothesized that fistula formation was initiated by microscopic leaks at the suture sites and that ADM would serve as an additional layer over the neo-urethra, covering the small gaps between sutures sites and reducing the incidence of fistula formation. The ADM constitutes a three-dimensional microskeleton that is replaced by autologous tissue after approximately 3 weeks. Autologous tissue contributes to reinforcement of periurethral tissue, further preventing the fistula formation [23].

We observed postoperative diverticulum in one patient $(2.9 \%)$ in the ADM group and in five patients $(6.25 \%)$ in the dartos group, which was similar to the results of prior studies. Vallasciani and associates [24] reported the incidence of postoperative diverticulum in $7 \%$ of patients after both onlay and tubularized preputial flap repairs during a mean follow-up 
period of 7 years; none of the patients had distal obstruction. Diverticulum is caused by a difference in urethral compliance between the native urethra and the neo-urethra. Accordingly, the urethra should be supported with several layers of tissue to reduce the difference in tissue elasticity. ADM retains the three-dimensional structure of the extracellular matrix, thereby regulating and guiding cell growth, promoting vascularization, and facilitating tissue regeneration [25]. When the collagen scaffold biomaterial was used as a urethral covering layer, it appears to provide extra tissue between the neourethral and penile skin. Therefore, the ADM group exhibited a lower incidence of diverticulum.

No glans dehiscence was observed in either group. Glans dehiscence typically occurs as a result of excessive tension in the tissue between the glans and the neo-urethra; alternatively, it may be caused by poor surgical technique. The average thickness of ADM is $0.30-0.69 \mathrm{~mm}$, which is similar to that of dartos; thus, there is no additional strain on the tissue [14]. Snodgrass and Bush [26] described complete dissection of glans wings from the corpora to 3 and 9 o'clock and release of the wings superiorly for approximately $4 \mathrm{~mm}$; they reported that this procedure successfully reduced the incidence of glans dehiscence.

The incidence of wound infection did not significantly differ between the ADM and dartos groups. This result was consistent with prior literature reports regarding proximal hypospadias repair, indicating that the use of this technique did not increase the incidence of adverse effects [19]. Although ADM is a foreign body, its structure rapidly degrades within host tissue. In previous studies, histological examinations of the extracellular matrix revealed complete replacement of the graft within 3 weeks [27]. Our previous study, comprising both clinical and experimental animal investigations, also showed that ADM exhibited good biocompatibility and low immunogenicity [12]. Infection rate was not statistically different between groups, but it was somewhat higher in the ADM treatment group. Further attention should be paid in the future large randomized comparative studies.
This preliminary study was limited in that it was conducted at a single center and enrolled a relatively small number of patients. Large randomized comparative studies are needed to assess the benefits of ADM when used alone or in addition to other tissue-covering approaches. Another limitation of this study was the relatively short follow-up time. Although most postoperative complications occur within the first half-year, future studies with longer followup are still required to evaluate the late complications.

\section{CONCLUSION}

We have successfully demonstrated a novel technique in which ADM served as the urethroplasty covering layer. Use of this biomaterial resulted in reduced incidence of urethrocutaneous fistula after urethroplasty in patients with severe hypospadias. This approach might constitute an effective strategy for hypospadias repair surgery.

\section{ACKNOWLEDGEMENTS}

Funding. This work, the Rapid Service and the Open Access Fees were supported by grants from the National Key R\&D Program of China (2016YFC 1000807, 2016YFC 1000801).

Authorship. All named authors met the International Committee of Medical Journal Editors (ICMJE) criteria for authorship for this manuscript, take responsibility for the integrity of the work as a whole, and approved for this version for publication.

Disclosures. Defu Lin, Guannan Wang, Hongcheng Song, Yanchao Qu, Pei Liu, Haiyan Liang, Shuai $\mathrm{Xu}$, Shuofan Chen, Weiping Zhang, Yannan Zhao, Bing Chen, and Ning Sun have nothing to disclose.

Compliance with Ethics Guidelines. The clinical study was approved by the ethics committee of Beijing Children's Hospital (IEC-C- 
006-A03-V.05). All procedures performed in studies involving human participants were performed in accordance with the ethical standards of Beijing Children's Hospital and with the 1964 Helsinki declaration and its later amendments or comparable ethical standards. Informed consent was obtained from parents to participate in the study and publish the results.

Open Access. This article is licensed under a Creative Commons Attribution-NonCommercial 4.0 International License, which permits any non-commercial use, sharing, adaptation, distribution and reproduction in any medium or format, as long as you give appropriate credit to the original author(s) and the source, provide a link to the Creative Commons licence, and indicate if changes were made. The images or other third party material in this article are included in the article's Creative Commons licence, unless indicated otherwise in a credit line to the material. If material is not included in the article's Creative Commons licence and your intended use is not permitted by statutory regulation or exceeds the permitted use, you will need to obtain permission directly from the copyright holder. To view a copy of this licence, visit http://creativecommons.org/licenses/by$\mathrm{nc} / 4.0 /$.

\section{REFERENCES}

1. Wong YS, Tam YH, Pang KKY, Yau HC. Incidence and diagnoses of disorders of sex development in proximal hypospadias. J. Pediatr. Surg. 2018;53: 2498-501.

2. Retik AB, Atala A. Complications of hypospadias repair. Urol Clin North Am. 2002;29:329-39.

3. Bhat A, Mandal AK. Acute postoperative complications of hypospadias repair. Indian J Urol. 2008;24: 241-8.

4. Yildiz A, Bakan V. Comparison of perimeatal-based flap and tubularized incised plate urethroplasty combined with single- or double-layer dartos flap in distal hypospadias. Urol Int. 2010;84:265-8.

5. Jia W, Liu G, Zhang L, et al. Comparison of tubularized incised plate urethroplasty combined with a meatus-based ventral dartos flap or dorsal dartos flap in hypospadias. Pediatr. Surg. Int. 2016;32: 411-5.

6. el-Kassaby AW, AbouShwareb T, Atala A. Randomized comparative study between buccal mucosal and acellular bladder matrix grafts in complex anterior urethral strictures. J. Urol. 2008;179: 1432-6.

7. Chun SY, Lim GJ, Kwon TG, et al. Identification and characterization of bioactive factors in bladder submucosa matrix. Biomaterials. 2007;28:4251-6.

8. Di Lullo GA, Sweeney SM, Korkko J, et al. Mapping the ligand-binding sites and disease-associated mutations on the most abundant protein in the human, type I collagen. J Biol Chem. 2002;277: 4223.

9. Jiang $\mathrm{P}$, Tang $\mathrm{X}$, Wang $\mathrm{H}$, et al. Collagen-binding basic fibroblast growth factor improves functional remodeling of scarred endometrium in uterine infertile women: a pilot study. Sci China Life Sci. 2019;62:1617-29.

10. Hou X, Shi C, Chen W, et al. Transplantation of human adipose-derived mesenchymal stem cells on a bladder acellular matrix for bladder regeneration in a canine model. Biomed Mater. 2016;11:031001.

11. Sumita Y, Honda MJ, Ohara T, et al. Performance of collagen sponge as a 3-D scaffold for tooth tissue engineering. Biomaterials. 2006;27:3238.

12. Jian G, Jianzhou L, Yuan G, et al. A myocardial patch made of collagen membranes loaded with collagen-binding human vascular endothelial growth factor accelerates healing of the injured rabbit heart. Tissue Eng Part A. 2011;17(21-22): 2739-47.

13. Chen W, Shi C, Yi S, et al. Bladder regeneration by collagen scaffolds with collagen binding human basic fibroblast growth factor. J Urol. 2010;183: 2432-9.

14. Mahmoud AY, Gouda S, Gamaan I, Baky F, Mohamed A. Autologous platelet-rich plasma covering urethroplasty versus dartos flap in distal hypospadias repair: a prospective randomized study. Int J Urol. 2019;26:475-80.

15. Bolland F, Korossis S, Wilshaw S-P, et al. Development and characterisation of a full-thickness acellular porcine bladder matrix for tissue engineering. Biomaterials. 2007;28:1061-70.

16. Van der Rest M, Garrone R. Collagen family of proteins. FASEB J. 1991;5(13):2814-23. 
17. Xu T, Zhang G, Bai W, et al. Complications and management of penile girth enhancement with acellular dermal matrix. J Sex Med. 2019;16:2011-7.

18. Asodiya FA, Kumar V, Vora SD, Singh VK, Fefar DT, Gajera HP. Preparation, characterization, and xenotransplantation of the caprine acellular dermal matrix. Xenotransplantation. 2019:e12572. https:// doi.org/10.1111/xen.12572.

19. Jia $\mathrm{W}$, Tang $\mathrm{H}, \mathrm{Wu}$ J, et al. Urethral tissue regeneration using collagen scaffold modified with collagen binding VEGF in a beagle model. Biomaterials. 2015;69:45-55.

20. Shi C, Chen W, Chen B, et al. Bladder regeneration in a canine model using a bladder acellular matrix loaded with a collagen-binding bFGF. Biomater Sci. 2017;5:2427-36.

21. Gopal SC, Gangopadhyay AN, Mohan TV, et al. Use of fibrin glue in preventing urethrocutaneous fistula after hypospadias repair. J. Pediatr. Surg. 2008;43:1869-72.

22. Abdol-Mohammad K, Shabnam S, Ali T, et al. The application of tissue-engineered preputial matrix and fibrin sealant for urethral reconstruction in rabbit model. Int Urol Nephrol. 2014;46(8): 1573-80.

23. Eppley BL. Experimental assessment of the revascularization of acellular dermis for soft-tissue augmentation. Plast Reconstr Surg. 2001;107:757-62.

24. Vallasciani S, Berrettini A, Nanni L, et al. Observational retrospective study on acquired megalourethra after primary proximal hypospadias repair and its recurrence after tapering. J Pediatr Urol. 2013;9:364-7.

25. Huang J-W, Xie M-K, Zhang Y, et al. Reconstruction of penile urethra with the 3-dimensional porous bladder acellular matrix in a rabbit model. Urology. 2014;84(6):1499-505.

26. Snodgrass W, Bush N. Is distal hypospadias repair mostly a cosmetic operation? J Pediatr Urol. 2018;14(4):339-40.

27. Kropp BP, Rippy MK, Badylak SF, et al. Regenerative urinary bladder augmentation using small intestinal submucosa: urodynamic and histopathologic assessment in long-term canine bladder augmentations. J Urol. 1996;155:2098. 